\title{
Clave ilustrada de los peces chilenos de la familia Serranidae (Teleostei: Perciformes)
}

\author{
Germán Pequeño ${ }^{1}$, José Rodrigo Rojas M. ${ }^{2} \&$ Sylvia Sáez ${ }^{1}$ \\ 1. Instituto de Zoología "Ernst F. Kilian” Universidad Austral de Chile, Casilla 567, Valdivia, Chile; \\ gpequeno@uach.cl; sylvia.saez@gmail.com \\ 2. Proceso de Planeamiento Ambiental, ICE, Costa Rica; yoyi66@yahoo.com
}

Recibido 0-I-2010. Corregido 0-VIII-2010. Aceptado 0-IX-2010

\begin{abstract}
Illustrated key for the Chilean fishes of the family Serranidae (Teleostei: Perciformes). A taxonomic key for identification of fish species of the family Serranidae of Chile, grouped in the subfamilies Epinephelinae, Serraninae and Anthiinae is presented. Basic data about the taxonomic of the group to facilitate the identification is given. In each specific case, the geographical distribution is showed. Reduced illustrations for the currently 13 serranid species considered in the Chilean ichthyofauna are included. Rev. Biol. Trop. 59 (1): 247-253. Epub 2011 March 01.
\end{abstract}

Key words: Taxonomy, Serranidae, South Eastern Pacific, Chile.

La familia Serranidae en Chile es actualmente considerada como un taxón que incluye tres subfamilias de peces óseos: Epinephelinae, que algunos autores consideran como familia, a partir de estudios filogenéticos (Smith \& Craig, 2007), Serraninae y Anthiinae, con una, tres y 10 especies, respectivamente (Rojas \& Pequeño, 1998, 2001). Estos peces viven de preferencia en las islas oceánicas chilenas y en la costa más septentrional de Chile continental, además revisten indudable importancia, por ejemplo, por sus interacciones con otros organismos, en el ambiente. Solo una especie, conocida como "cabrilla común" Paralabrax humeralis (Valeneciennes, 1828), está incluida en las estadísticas pesqueras de Chile (SERNAP 2000), pese a que es un hecho conocido que varias especies tales como por ejemplo Acantisthius fuscus Kendall \& Radcliffe, 1912 y A. pictus (Tschudi, 1845), son transadas en el comercio en Isla de Pascua y en el norte de Chile, respectivamente. En cambio, en estas estadísticas aparece el "apañado" Hemitlutjanus macrophthalmos
(Tschudi, 1844), como perteneciente a la familia Serranidae. Cabe comentar que el género Hemitlutjanus Bleeker, 1876, es considerado monotípico, de ubicación incierta y no un lutjánido (Fam. Lutjanidae) (Johnson 1984, Eschmeyer 1998). En la actualidad, se estima que $H$. macrophthalmos es una especie de dudosa ubicación taxonómica (insertae sedis), aunque se incluye dentro del Suborden Percoidei del Orden Perciformes, sin precisar familia ni otros taxa menores, donde pudiese estar clasificada (Eschmeyer 1998). Sin embargo, estudios recientes, la ubican a la especie en la Subfamilia Anthiinae, de la familia Serranidae (Chirichichigno \& Vélez 1998). En este trabajo se ha optado por seguir el criterio de Eschmeyer (loc. cit.).

Las especies de Serranidae de Chile han sido revisadas hace poco tiempo, pero en forma separada, con fines principalmente sistemáticos y zoogeográficos (Rojas \& Pequeño 1998, 2001). Sin embargo, dada la importancia, tanto del grupo como de cada una de las especies que lo integran, es evidente la necesidad de poder 
contar con un sólo instrumento para reconocer con el máximo posible de rapidez y en terreno, los taxa mencionados. Para cumplir tal objetivo, se ha preparado el presente trabajo.

\section{MATERIAL Y MÉTODOS}

Los trabajos de Rojas \& Pequeño (1998, 2001) así como otras contribuciones (De Buen 1959, Anderson \& Baldwin 2002) han servido de base para la preparación del presente aporte y tanto los materiales como los métodos, son los mismos descritos por Rojas \& Pequeño (1998, 2001).

Para facilitar la comprensión de algunas características externas mencionadas en la clave, se sugiere consultar las Figs. 1 y 2.

\section{RESULTADOS}

El análisis de la familia Serranidae de los litorales de Chile, permite reordenar taxonómicamente las especies de esta región en la siguiente forma.

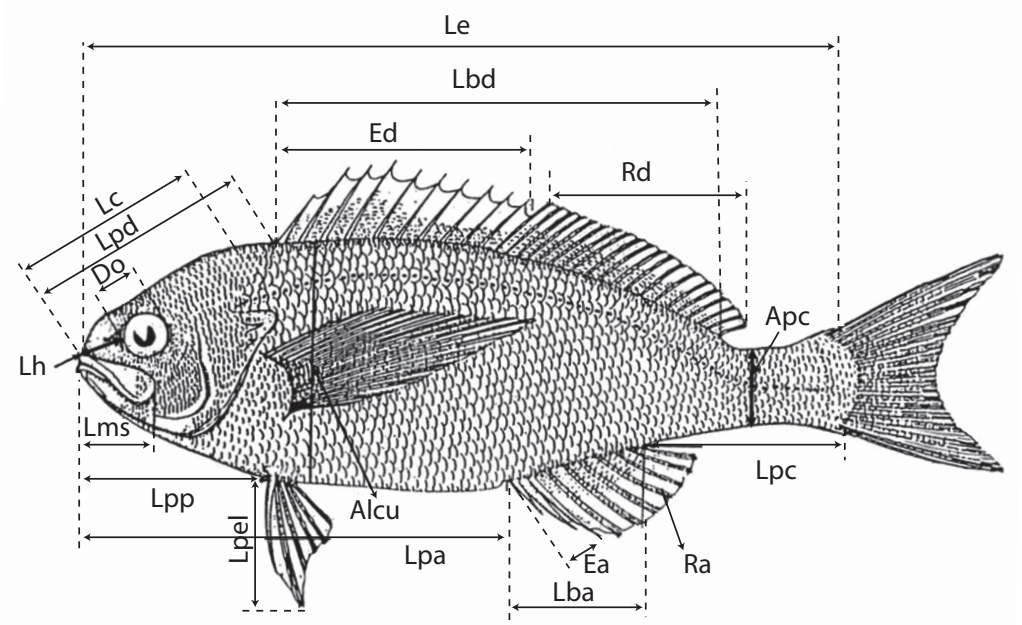

Fig. 1. Atributos morfológicos considerados en el reconocimiento de la serranifauna chilena. (Rojas \& Pequeño 1998).

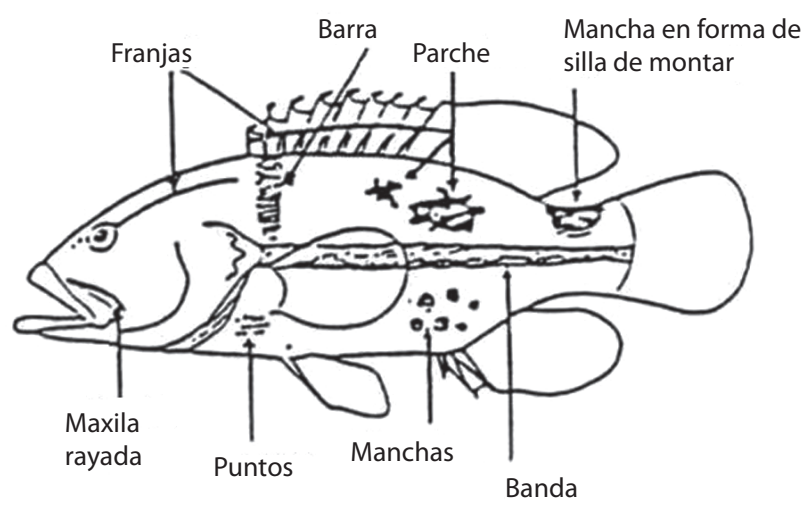

Fig. 2. Patrones de coloración utilizados en la clave taxonómica. (Rojas \& Pequeño 1998). 


\section{Clave comprensiva e ilustrada para peces de la Familia Serranidae de Chile}

1 (2) Escamas del cuerpo pequeñas, más de 80 en serie oblicua desde el extremo superior de la abertura branquial hasta la base de la aleta caudal; escamas de la línea lateral discretas, poco notorias, por lo general más pequeñas que las escamas del cuerpo y en su mayor parte cubierta por ellas; repliegue de la piel con escamas junto a la parte superior de la base de la aleta pectoral al cuerpo . . . . . . . . . . . . . . . . . . EPINEPHELINAE - Menos de ocho espinas dorsales (siempre siete), siempre más de ocho radios anales, línea lateral incompleta, menos de 15 pseudobranquias, más de 19 radios dorsales. (Isla de Pascua) Pseudogramma australis pasquensis Randall \& Baldwin, 1997. (Fig. 3)

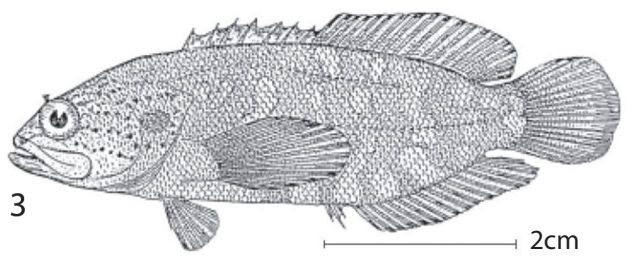

2 (1) Escamas del cuerpo más grandes, generalmente menos de 80 en serie oblicua desde el extremo superior de la abertura branquial hasta la base de la aleta caudal; escamas de la línea lateral diferentes, subiguales en tamaño y en número de las hileras adyacentes repliegue de la piel con escamas junto la parte superior de la base de la aleta

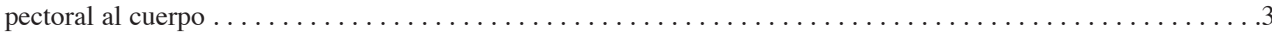

3 (4) Maxila desnuda; escamas de la línea lateral $66-77 \ldots \ldots \ldots \ldots \ldots \ldots \ldots \ldots \ldots \ldots$ SERRANINANE...5

4 (3) Maxila con escamas en la mayoría de las especies; de 28-60 escamas en la línea lateral . . . . .ANTHINAE...9

5 (6) Menos de 60 escamas tubulares en la línea lateral, menos de 30 pseudobranquias, menos de 15 escamas entre la línea lateral y la base de inserción de la espina anal, menos de 60 dentículos en el preopérculo. . . . . . . . .7

6 (5) Más de 60 escamas tubulares en la línea lateral, más de 30 pseudobranquias, más de 15 escamas entre la línea lateral y la base de inserción de la espina anal, más de 60 dentículos en el preopérculo. (Desde la Rada Quintay, Valparaíso hasta Colombia) ................. Paralabrax humeralis (Valenciennes, 1828). (Fig. 4)

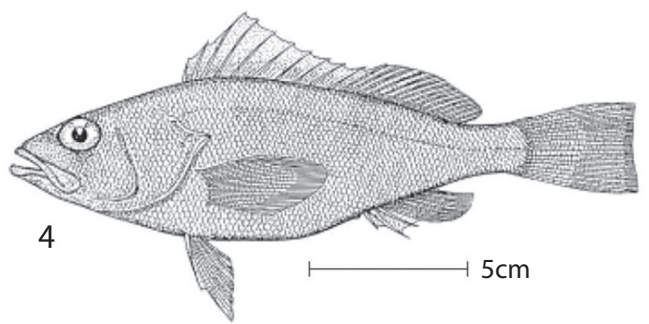

7 (8) Más de 25 branquispinas en el primer arco branquial (incluyendo rudimentos); la tercera espina dorsales es más larga; tercera espina anal la más gruesa y larga; un grueso parche de dentículos presente en el preopérculo, que llega a formar una verdadera espina, es decir, la sierra ubicada en el ángulo preopercular. (Desde la Bahía de Concepción, Chile hasta las costas ecuatorianas) ......... Diplectrum conceptione Valenciennes, 1828. (Fig. 5)

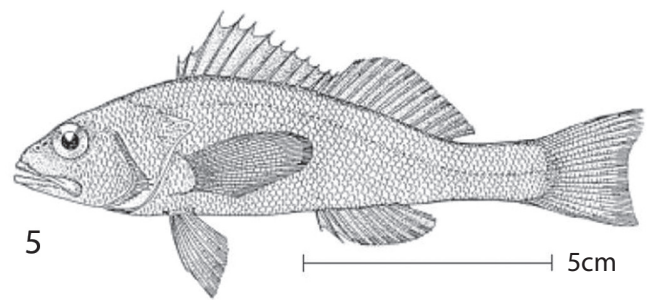


8 (7) Menos de 25 branquispinas en el primer arco branquial (incluyendo rudimentos); sexta espina dorsal es la más larga, segunda espina anal es la más gruesa y larga; los dentículos del preopérculo no forman un grueso parche. (Desde el Golfo de California hasta el centro-norte de Chile). . . Serranus huascarii Steindachner, 1900. (Fig. 6)

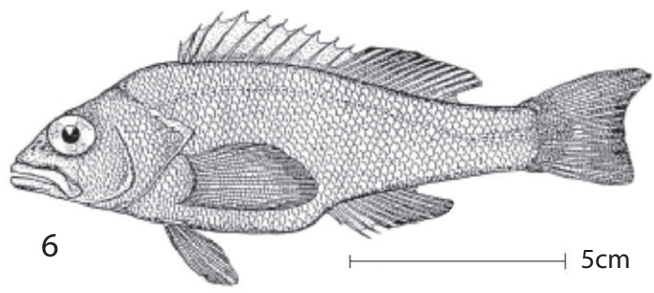

9 (10) Premaxilar, dentario, palatinos y vómer con una delgada banda de dientes villiformes (siempre menos de 3 filas de dientes), aleta caudal truncada o bifurcada, distancia preopercular mayor al 5\% de la longitud estándar . . . . 11

10 (9) Premaxilar, dentario, palatinos y vómer con una gruesa banda de dientes viliformes (siempre más de tres filas de dientes), aleta caudal redondeada convexa, distancia preopercular menor al 5\% de la longitud estándar. (Islas Norfolk (localidad tipo), Australia, Nueva Zelandia, Isla Kermadec, Isla de Pascua e Islas Desventuradas) Trachypoma macracanthus Günther, 1859. (Fig. 7)

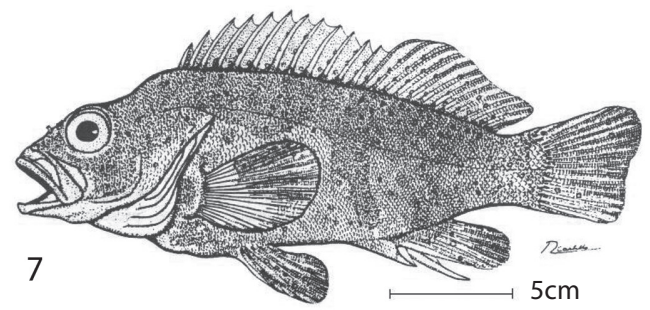

11 (12) Menos de 21 radios pectorales, mandíbula no abruptamente expansible, más de 18 pseudobranquias, más de 12 branquiespinas en el primer arco branquial (incluye rudimentos), longitud cefálica, más del $28 \%$ de la longitud

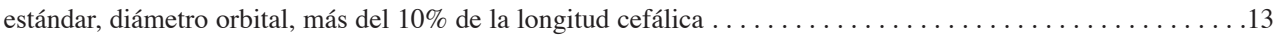

12 (11) 21 radios pectorales, mandíbula abruptamente expansible, menos de 18 pseudobranquias, menos de 12 branquispinas en el primer arco branquial (incluyendo rudimentos), longitud cefálica, menos del $28 \%$ de la longitud estándar, diámetro orbital, menos del $10 \%$ de la longitud cefálica. (frente a la costa centro-norte de Chile, cerca del extremo suroeste de la Cordillera sumergida de Nazca) Anatolanthias apiomycter Anderson, Parin y Randall, 1999. (Fig. 8)

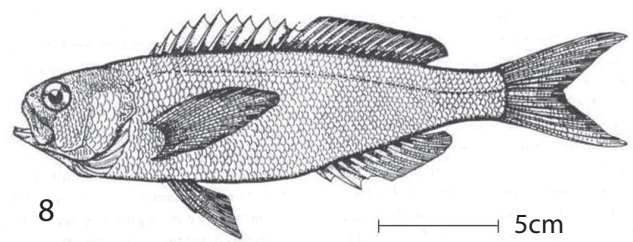

13 (14) Menos de 90 aserraciones en el preopérculo, tercero, cuarto y quinto radios pélvicos no fusionados, último radio dorsal no alargado; radios caudales no fusionados; menos de 33 branquiespinas; longitud de aleta pélvica menos del $35 \%$ de la longitud estándar, longitud de la aleta anal menos del $20 \%$ de la longitud estándar . . . . . . .15

14 (13) Más de 90 aserraciones en el preopérculo, tercero, cuarto y quinto radios pélvicos fusionados, último radio dorsal alargado; radios caudales no fusionados; más de 33 branquiespinas; longitud de aleta pélvica más del 35\% de la longitud estándar; longitud de la aleta anal menos del 20\% de la longitud estándar. (Desde la costa oeste de Baja California Sur y Golfo de California (México) posiblemente hasta Antofagasta (Chile)) Hemanthias peruanus Steindachner, 1874. (Fig. 9) 


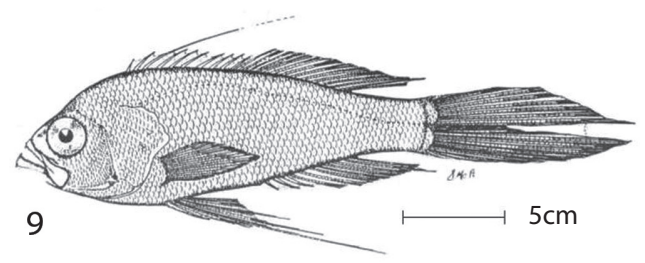

15 (16) Más de 10 espinas dorsales, más de 90 escamas en filas sobre la línea lateral . . .

16 (15) 10 o menos espinas dorsales, menos de 90 escamas en filas sobre la línea lateral .

.19

17 (18) 11 espinas dorsales; distancia interorbital con más de 6 escamas; distancia suborbital con más de cinco escamas; una espina antrorsa en el borde ventral del preopérculo; menos de 60 pseudobranquias; la branquiespina más larga no está en el ángulo del primer arco branquial. (Zona norte de Chile (Antofagasta, Iquique y Arica) y Sur del Perú) Acanthistius pictus (Tschudi, 1845). (Fig. 10)

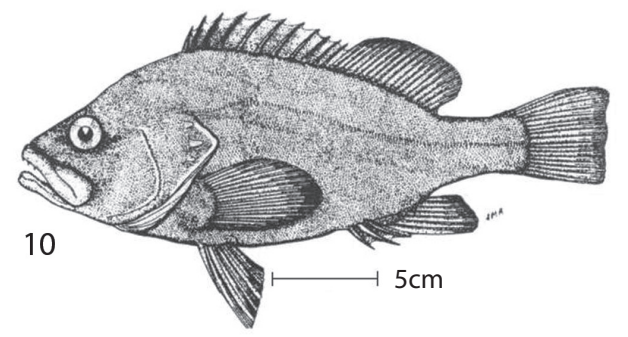

18 (17) Más de 11 espinas dorsales; distancia interorbital con menos de seis escamas; distancia suborbital con menos de cinco escamas; más de una espina antrorsa en el borde ventral del preopérculo; más de 60 pseudobranquias; la branquisepina más larga no está en el ángulo del primer arco branquial. (Isla de Pascua e isla Salas y Gómez) Acanthistius fuscus Regan, 1913. (Fig. 11)

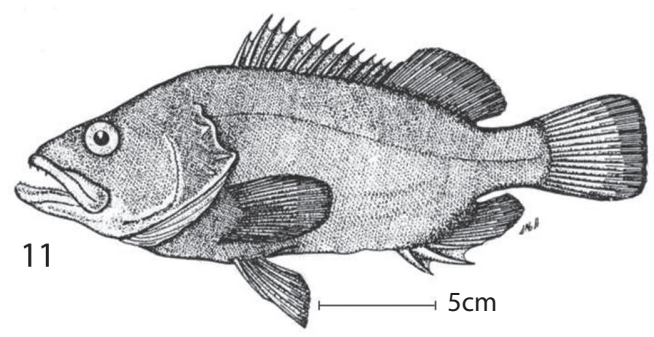

19 (20) 26 o menos vértebras; menos de seis escamas entre la primera espina dorsal; bandas verticales oscuras entre la base

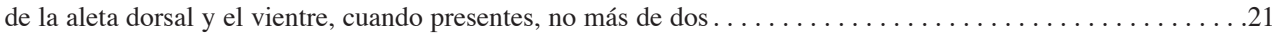

20 (19) Más de 26 vértebras (por lo general 27 ó 28 vértebras muy rara vez 26); más de seis escamas entre la primera espina dorsal; bandas verticales oscuras entre la base de la aleta dorsal y el vientre, cuando existen, más de dos (comúnmente entre siete u ocho). (Archipiélago de Juan Fernández, Islas Desventuradas e Isla de Pascua). Hypoplectrodes semicinctum Valenciennes, 1833. (Fig. 12)

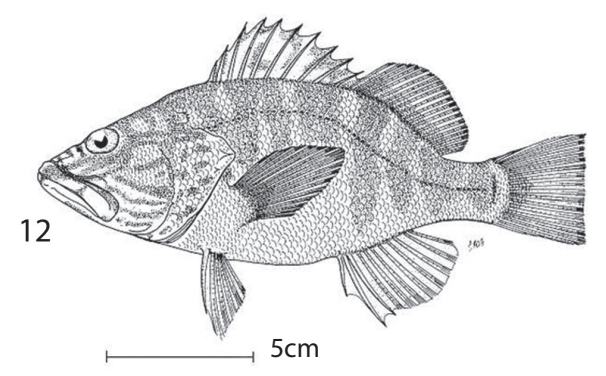


21 (22) Menos de 35 pseudobranquias; branquiespinas menores, iguales o ligeramente mayores a los filamentos branquiales, segundo radio dorsal y radios caudales dorsales superan en longitud la espina dorsal más larga, aleta caudal redondeada convexa o truncada, interopérculo y subopérculo con aserraciones $\ldots \ldots \ldots \ldots \ldots \ldots \ldots \ldots$

22 (21) Más de 35 pseudobranquias; branquiespinas más largas que los filamentos branquiales; segundo radio dorsal y radios caudales dorsales no superan en longitud a la espina dorsal más larga; aleta caudal ahorquillada; interopérculo y subopérculo sin aserraciones. (Australia, Nueva Zelandia, Nueva Gales del Sur, Islas Hawaianas, Formosa, Corea, Sur de Japón, Isla Midway, Isla Lord Howe, Isla Tuamotu, Isla Kermadec, Isla de Pascua, Juan

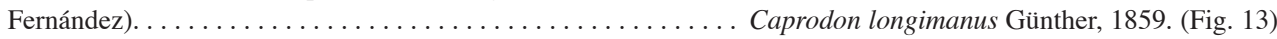

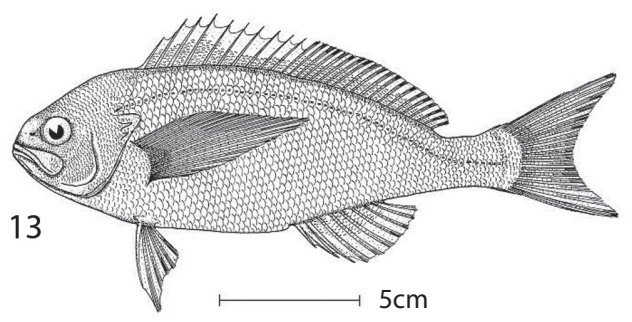

23 (24) Más de 27 pseudobranquias; 10 o más filas de escamas en las mejillas; por lo general más de 25 pseudobranquias; más de 17 escamas entre la línea lateral y el origen de la aleta dorsal; maxilar alcanza escasamente el nivel del primer cuarto de la órbita; aleta caudal con radios procurrentes (dorsal y ventral) y provistos de escamas; más de 40 escamas tubulares en la línea lateral; longitud cefálica, menos del 40\% de la longitud estándar (Archipiélago de Juan Fernández, Islas Desventuradas y Cordillera Sumergida de Nazca)

Plectranthias exsul Heemstra \& Anderson, 1983. (Fig. 14)

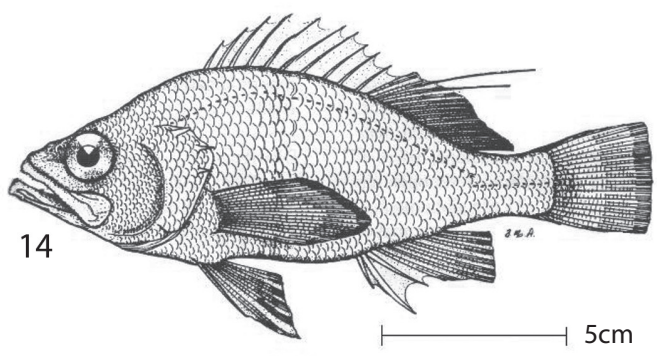

24 (23) Menos de 27 pseudobranquias; menos de 10 escamas en las mejillas, no más de 25 pseudobranquias; menos de 17 escamas entre la línea lateral y el origen de la aleta dorsal; el maxilar alcanza más allá del nivel del primer cuarto de la órbita ocular; aleta caudal con radios procurrentes (dorsales y ventrales) desprovistos de escamas; menos de 40 escamas tubulares en la línea lateral, longitud cefálica, mayor del 40\% de la longitud estándar: (Cordillera sumergida de Salas y Gómez (2700 km al oeste de la bahía de Valparaíso))

Plectranthias parini Anderson \& Randall, 1991. (Fig. 15)

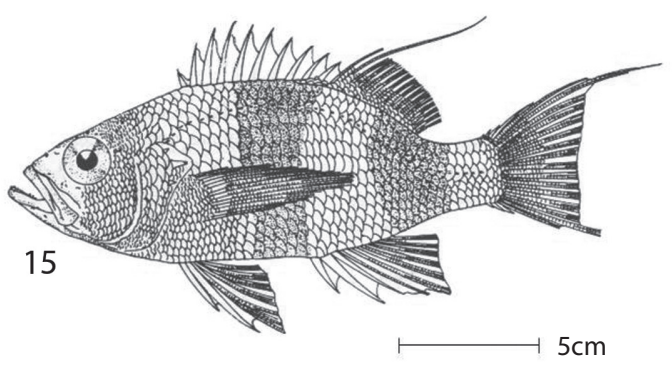




\section{DISCUSIÓN}

En la actualidad, se consideran para Chile 13 especies agrupadas en 11 géneros pertenecientes a tres subfamilias de la familia Serranidae. Hace algunos años, se describió la especie Plectranthias lamillai Rojas \& Pequeño 1998 y poco tiempo después fue desestimada y considerada en la sinonimia de Plectranthias exsul Heemstra \& Anderson, 1983. Entre las características anotadas para $P$. lamillai se señaló un patrón de coloración no reconocido para $P$. exsul, pero será necesario conseguir material adicional para continuar estudiando este asunto.

De las 13 especies consideradas cinco habitan solo la costa continental de Chile y dos se distribuyen hasta Baja California Sur y Golfo de California, Diplectrum conceptione alcanza la latitud más austral (la bahía de Concepción). Las otras siete especies se localizan en las islas oceánicas chilenas y en las cordilleras sumergidas de Nazca y de Salas y Gómez; y dos son reconocidas como anfipacíficas (Trachypoma macracanthus y Caprodon longimanus) pues ambas se conocen en Australia, Nueva Zelandia y diferentes localidades de océano Pacífico suroccidental.

\section{AGRADECIMIENTOS}

Se agradece la colaboración de León Matamala M. (Instituto de Zoología, Universidad Austral de Chile), así como el apoyo al proyecto S-199911 de la Dirección de Investigación y Desarrollo (DID), de la misma Universidad y al Proyecto CIMAR-5 Islas, del Comité Oceanográfico Nacional (CONA), al primer autor. La Deutscher Akademischer Austauschdienst (DAAD) de la República Federal de Alemania prestó valioso apoyo al segundo autor.

\section{RESUMEN}

Se presenta una clave para reconocer especies de la familia Serranidae de Chile, que se agrupan en las subfamilias Epinephelinae, Serraninae y Anthiinae. Se proporcionan antecedentes básicos referidos a la taxonomía del grupo, para mayor facilidad del reconocimiento. En cada caso específico, se indica la distribución geográfica e ilustraciones reducidas de las 13 especies de la familia Serranidae que en la actualidad son consideradas en la ictiofauna de Chile.

Palabras clave: Taxonomía, Serranidae, Pacífico Suroriental, Chile.

\section{REFERENCIAS}

Anderson, W. Jr. \& C.C. Baldwin. 2002. Plectranthias lamillai Rojas and Pequeño, 1998: A junior Synonym of Plectranthias exsul Heemstra and Anderson, 1983. Copeia 2002: 233-238.

Chirichigno, F.N. \& J. Vélez. 1998. Clave para identificar los peces marinos del Perú. Publicación Especial, Instituto del Mar del Perú.

De Buen, F. 1959. Lampreas, tiburones, rayas y peces de la Estación de Biología Marina de Montemar, Chile. Rev. Biol. Mar., Valparaíso 9: 1-200.

Eschmeyer, W.N. 1998. Part II, Genera of Fishes, pp. 1821-2174. In W.N. Eschmeyer (ed.). Catalog of Fishes Vol. 3, California Academy of Sciences, San Francisco, EEUU.

Johnson, G.D. 1984. Percoidei: development and relationships. In HG Moser, W.J. Richards, D.M. Cohen, M.P. Fahay, A.W. Jr. Kendall \& S. Richardson (eds). Ontogeny and systematics of fishes. American Society of Ichthyologists and Herpetologists, Special Publication No. 1: 464-498.

Rojas, J.R. \& G. Pequeño. 1998. Revisión taxonómica de los peces subfamilia Anthiinae del Pacífico suroriental chileno (Pisces: Serranidae: Anthiinae). Rev. Biol. Mar. y Oceanogr. 33: 163-198.

Rojas J.R. \& G. Pequeño. 2001. Revisión taxonómica de especies de las subfamilias Epinephelinae y Serraninae (Pisces: Serranidae) de Chile. Rev. Biol. Trop. 49: 157-171.

SERNAP (Servicio Nacional de Pesca). 2000. Anuario Estadístico de Pesca. Ministerio de Economía, Fomento y Reconstrucción, Chile.

Smith W.L. \& M. Craig. 2007. Casting the percomoph net widely: the importance of broad taxonomic sampling in the search for the placement of serranid and percid fishes. Copeia 2007: 35-55. 